\title{
(2) \\ Registration of premises and inspections
}

\section{KEY POINTS}

- Veterinary surgeons (veterinarians) can only supply veterinary medicines from registered veterinary practice premises (VPP)

- Inspectors will check Controlled Drugs (CDs) records, storage of medicines, medicines' records, supply procedures and disposal of medicines

- All VPP will have a medicines inspection either from the Royal College of Veterinary Surgeons (RCVS) or the Veterinary Medicines Directorate (VMD)

- There will be a report after the inspection setting out any action points or deficiencies found, which will have to be corrected

n The VMD is responsible for enforcement of the Veterinary Medicines Regulations (VMR) and can issue improvement and seizure notices

\section{Registration of veterinary practice premises}

A veterinary surgeon may only supply veterinary medicinal products (VMP) classified as 'prescriptiononly medicine - veterinarian' (POM-V), 'prescriptiononly medicine - veterinarian, pharmacist, suitably qualified person (SQP)' (POM-VPS), 'non-food animal - veterinarian, pharmacist, SQP' (NFA-VPS) or cascade products from premises registered with the RCVS as a VPP. Application forms for the registration of a VPP are available to download from the RCVS website (@)

Completed forms and the registration fee (currently £34) must be sent to the RCVS registration department. On receipt of a completed application, the RCVS will process the form and add the premises
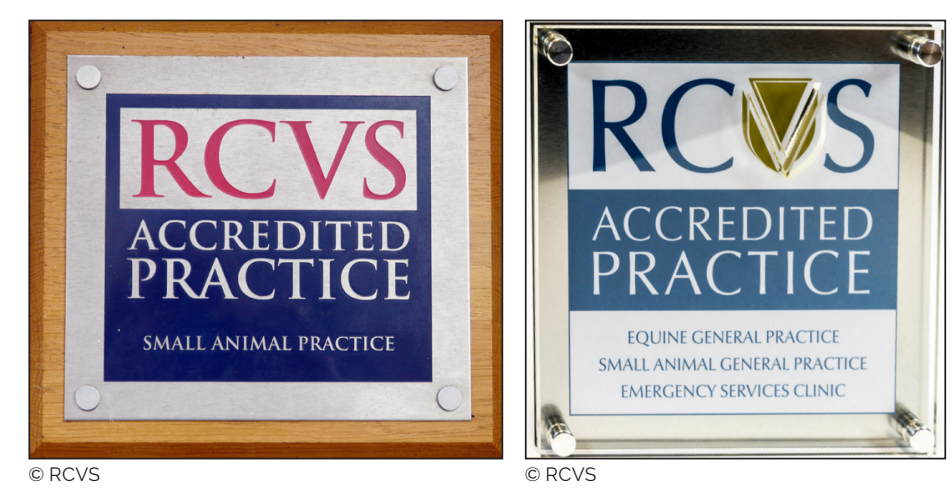
to the Register of VPP (RVPP). The RCVS holds the RVPP on behalf of the VMD.

Practices that are accredited members of the RCVS Practice Standards Scheme (PSS) do not have to register separately, as the RCVS already holds sufficient information on them to fulfil the registration requirements. On a regular basis, the RCVS provides the VMD with details of non-PSS VPP.

The requirement to register a VPP relates to vets supplying from registered practice premises, and does not mean that every veterinary surgeon must have a registered VPP of their own. The provisions create a register of VPP, not a register of veterinary surgeons.

\section{Inspection of VPP}

Inspections of registered VPP are regularly carried out to assess compliance with the VMR, and the Misuse of Drugs Regulations 2001.

Inspections generally focus on:

- How medicines are prescribed and supplied

- Storage of veterinary medicines, particularly temperature-sensitive products such as vaccines

- Storage and recording of Controlled Drugs (CDs)

- Record keeping for POM-V, POM-VPS and cascade products.

Veterinary practices that are members of the PSS are inspected by the RCVS PSS assessors and a medicines inspection forms part of the whole practice inspection.

PSS practices have a full assessment every 4 years. When a PSS practice is due for an assessment, the PSS team will contact the practice via Stanley (the RCVS PSS administration tool) with details of the proposed assessor. If the practice 
perceives a conflict of interest with the assessor, they can request a different assessor. The assessor will then contact the practice to arrange a mutually agreeable date for the visit, which must take place within 3 months. Practices can also be notified of a spot check at any point with 24-48 hours' notice. These are short 1-2 hour assessments and focus on limited areas, which may include medicines.

Practices pay an application fee to join the PSS and then an annual fee based on the number of individual premises. An assessment fee is only charged if an assessment is requested by the practice because of moving premises or changing accreditation. An additional fee is also charged if the practice decides to be assessed for the optional awards.

The VMD aims to inspect non-PSS practice premises at least once every 4 years, and charges a fee for each inspection. The frequency depends on the level of compliance found at the previous inspection (see below). The VMD will generally give a VPP at least 10 days' written notice of an intended inspection. The notification letter explains what the VMD will check and points the practice to the inspection criteria in the retail of veterinary medicines guidance (formerly Veterinary Medicines Guidance Note Number 3 Annex B) (@)

If a VPP has applied to the PSS and provides the VMD with written confirmation from the RCVS, the VMD will cancel its inspection. In all other cases, the VMD will only agree to cancel an inspection if there is a genuine and justified request from a VPP, and the VPP proposes an alternative date. If the VPP does not confirm a date for another inspection, the VMD will make an unannounced inspection within 4 months.

If a VMD inspector arrives to conduct an inspection but is unable to because no-one is available, the VMD will charge an appropriate amount of the fee for the visit.

The VMD also inspects SQP retailer premises, and they must be inspected before they can be approved. Applicants should allow 10 days for the application to be processed and then a further 30 working days for an inspection. They will then be notified if their premises are suitable to be approved and receive written confirmation. SQP retailers are notified a few days in advance of an inspection. SQPs should be aware of the inspection criteria as they are repeated in their Code of Practice.

\section{The on-site inspection}

The inspection criteria are set out on the 'retail of veterinary medicines' page of the VMD website. A summary of the general inspection findings is also published on the VMD website on the 'registration and inspection of veterinary practice premises' page (@)).

The RCVS PSS criteria and standards are set out in the Practice Standards Scheme Small Animal Modules and Awards document (@). Section 8 (Medicinal Products) covers Medicines and the Dispensary area.

Both the VMD and the PSS inspections include checks on the RCVS registration of veterinary surgeons and premises, as well as the registration and qualifications of any SQPs. (9) See also Suitably Qualified Persons.

On arrival, the VMD inspector will explain the purpose of the inspection, and ask about the practice. They will want to know, for example:

- The main contact people (and email addresses)

- What species the practice treats (e. g. companion animal only, equine, mixed)

- The number of veterinary surgeons and veterinary nurses

- What practice management system is in place

Who the wholesale supplier is.

RCVS PSS assessors will expect to speak to a cross-section of staff involved in the normal activities of an operational day. The purpose of such discussions is so that the assessor can be satisfied that practice policies are not only in place but are understood by relevant staff and are applied in the day-to-day operation of the practice, and to encourage better practice. The assessor will record the number of veterinary surgeons, veterinary nurses and other members of staff spoken to in the course of an inspection.

Both RCVS assessors and VMD inspectors will cover the inspection criteria. In relation to the supply of VMP, they will particularly check the following aspects:

\section{Premises}

- The premises are a permanent and secure building or a mobile unit based at a fixed address, which does not allow the entrance and harbouring of wild birds or vermin.

- There are appropriate staff amenities, toilets and hand washing facilities, and these are separate from the VMP storage areas.

\section{Controlled Drugs}

- CDs are appropriately stored and recorded, and in the case of Schedule 2 CDs, they are being continuously audited (9) See also Controlled Drugs.

\section{Storage of medicines}

- The storage arrangements allow VMP to be stored in accordance with the manufacturers' recommendations in the summary of product characteristics (SPC). Storage areas need to be monitored by the use of maximum and minimum thermometers to check that temperatures do not fluctuate from the manufacturers' recommended range, and the maximum/minimum temperatures are regularly recorded.

- VMP are stored in areas away from excessive light/moisture.

- VMP are stored in areas that are not accessible to the public or domestic pets. 
- There are no VMP on self-service, except authorized veterinary medicines - general sales list (AVM-GSLs) or those marketed under the Schedule 6 exemption.

- There is an effective stock control system in operation and out of date, damaged or returned medicines are segregated from usable products and ultimately disposed of correctly.

(9) See also Medicine waste disposal.

(9) See also Correct storage, dispensary management and standard operating procedures.

\section{Records}

Appropriate records are kept of all VMP, and in particular those supplied under the cascade and for food producing animals.

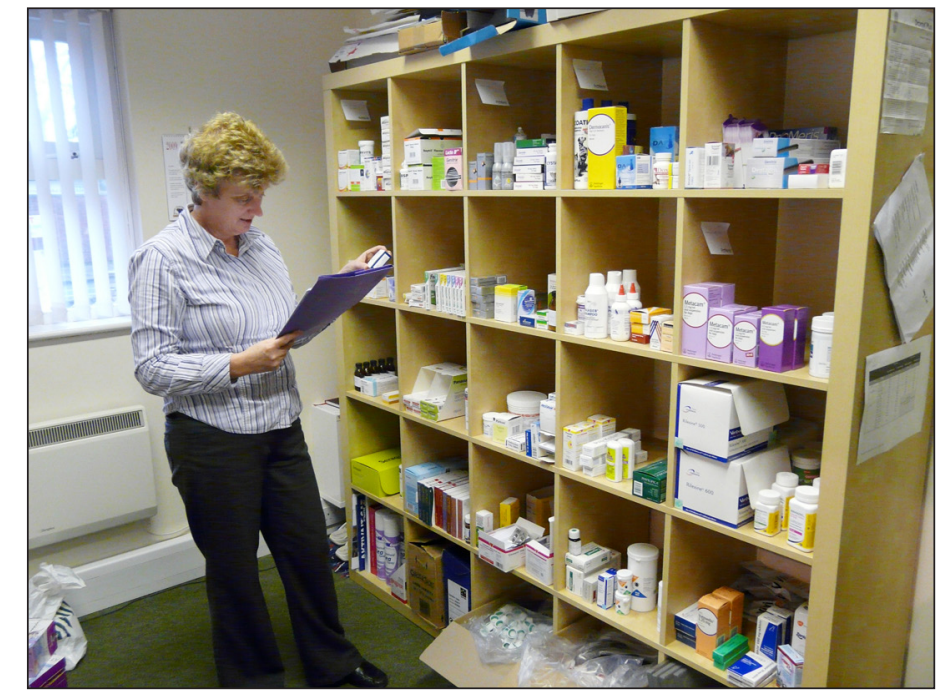

(9) See also Record keeping and audits.

\section{Supply procedures}

- Out of date VMP, including those past their 'broached or use-by' date, are not used or supplied to clients (it is illegal to use or supply a product after its expiry date).

- For POM-V medicines supplied, there is evidence that they have been prescribed by a veterinary surgeon, and that each transaction has been authorized by a veterinary surgeon or pharmacist. In practice, this may be achieved by:

- The veterinary surgeon handing over the medicine

- The veterinary surgeon making a note on the client's record that repeat prescriptions can be supplied for a set time

- A member of staff taking a telephone request from a client and getting the veterinary surgeon to authorize the medicine before it is supplied.

- For POM-VPS medicines supplied, there is evidence that they have been prescribed by a veterinary surgeon, pharmacist or SQP, and that each transaction has been authorized by one of those professionals.

- For POM-V, POM-VPS and NFA-VPS medicines, there is evidence that before the medicine is supplied, the prescriber:

- Checked that the person who will be administering the medicine is competent to do so safely

- Checked that the client intends to use it for a purpose for which it is authorized

- Advised the client on safe administration of the medicine and on any warnings/contraindications stated on the label or package leaflet.

- Staff 'handing over' medicines to clients have been trained to do so (e. g. have knowledge of practice protocols and have a standard operating procedure (SOP) in place). Both VMD inspectors and PSS assessors will expect to talk to staff to ascertain how training takes place.

- All veterinary medicines, including medicines prescribed in accordance with the cascade, are appropriately labelled (9) See also Prescribing, supplying, dispensary and labelling procedures.

- Written prescriptions include all the information required under the VMR.

See also Correct storage, dispensary management and standard operating procedures.

\section{Disposal}

- Unusable VMP are appropriately disposed of and recorded (e.g. by logging disposed products against a 'dummy' client on the practice management system called 'Disposal').

See also Medicine waste disposal.

\section{Reporting the results of the inspection}

The VMD inspector will summarize the inspection findings at the end of the inspection. They will then send a written report within 4 weeks, which details the findings and corrective actions required (if any). The VPP will also be invoiced for the inspection.

PSS assessors will submit a report to the RCVS within 14 days. This will recommend one of the following:

- Outright pass or fail

- Pass subject to compliance with stated conditions within a stipulated timeframe

- Pass at a category other than that applied for.

The action points will be shown on the report. If there are large numbers of action points, a re-assessment may be necessary. Normally, the practice will be able to upload evidence on to Stanley to show that they have complied with the action points. There is a timeframe for evidence upload. For Controlled Drugs, or other serious issues, the timeframe is usually 1 month; for other medicines it is usually 3 months.

In the case of VMD inspections, the deficiencies are classified as minor (other), major or critical. 


\section{Minor (other) deficiencies}

- A deficiency that is minor and poses no potential risk to human or animal health, or the environment.

- A deficiency that does not indicate a significant deviation from the requirements of the VMR, Codes of Practice or Guidance.

- A deficiency that cannot be classified as either critical or major, because there is insufficient information to classify it as such.

\section{Major deficiencies}

- A non-critical deficiency that has produced, or has the potential to produce, a possible risk to human or animal health, or the environment.

- A deficiency that indicates a major deviation from the requirements of the VMR.

- A deficiency that indicates a failure to carry out satisfactory procedures to ensure that products are manufactured, stored or distributed in accordance with their specific requirements

- A combination of more than six minor (other) deficiencies, none of which on their own may be major, but which may together represent a major deficiency and should be explained and reported as such.

- Minor (other) deficiencies that have been brought to the attention of the business on previous occasions but have not been resolved.

\section{Critical deficiencies}

- Deficiencies that have produced, or have the potential to produce, a significant risk to human or animal health, or the environment.

- A deficiency that indicates a significant deviation from the requirements of the VMR through serious negligence or intent.

The VMD then applies an inspection interval based on the number and type of deficiencies noted, as follows:

\section{VMD Veterinary Practice Premises risk-based inspection plan}

\begin{tabular}{|l|l|l|l|}
\hline Inspection findings & $\begin{array}{l}\text { Compliance } \\
\text { category }\end{array}$ & Inspection points* & $\begin{array}{l}\text { Max. inspection interval in } \\
\text { months by category }\end{array}$ \\
\hline 0 deficiencies; recommendations only & 5 & 0 & 48 \\
\hline 1-6 minor (other) & 4 & $1-6$ & 48 \\
\hline $\begin{array}{l}\text { More than } 6 \text { minors and/or 1-3 majors } \\
\text { 3 majors plus 1 or more minors up to and } \\
\text { including 5 majors }\end{array}$ & 3 & $7-21$ & 36 \\
\hline More than 5 majors and/or any critical & 2 & $22-35$ & 24 \\
\hline
\end{tabular}

*A minor deficiency = 1 point; a major deficiency = 7 points; a critical deficiency = 36 points

\section{Enforcement}

The VMD remains responsible for enforcement of the VMR in all VPP. The VMD inspectors follow an Enforcement Strategy (i) that aims to deal with deficiencies (non-compliance) by taking an 'inform, insist, enforce' approach.

\section{Improvement notices}

The VMD inspectors have the power to serve improvement notices on any person they believe is not complying with the VMR. The notice will clearly set out:

- How that practice/person is failing to comply with the VMR

- The exact nature of the failure

- Measures that need to be taken to comply

- How quickly these measures should be taken.

All improvement notices will give the person at least 14 days to take the measures necessary to comply with the VMR.

\section{Seizure notices}

The VMD inspectors also have the power to seize products, records and equipment (including computers) when they believe those items are related to a serious breach of the VMR (critical deficiencies). The seizure notice will set out the inspector's grounds for seizing the item(s) and inform the practice/person how they can make a claim against the seizure. If no claim is made within 28 days then, unless the products are being retained for criminal proceedings, they will be destroyed.

- In the most serious of breaches, the VMD may instruct the RCVS to remove a premises from the register of VPP.

- In the formal actions outlined above, there are appeal procedures set out in the VMR.

- An individual or company may also be prosecuted.

- All improvement notices, seizure notices and prosecutions are published on the VMD website and in its quarterly online journal, Marketing Authorisation Veterinary Information Service (MAVIS). 


\section{QUESTIONS}

1. A veterinary surgeon can supply POM-V, POM-VPS, NFA-VPS and cascade medicines:
a. Only from a veterinary practice premises registered with the RCVS
b. From any veterinary practice, pharmacy or SQP retailer premises
c. Only from a premises that the VMD have inspected
d. Only from their own registered premises

2. The standard interval between inspections is:
a. 5 years
b. 5 years for PSS and 4 years for the VMD
c. 4 years
d. 4 years for PSS and 2 years for VMD

3. Inspectors/Assessors will check that for POM-V and POM-VPS medicines there is evidence that the prescribing veterinary surgeon has:

a. Checked that the person who will be administering the medicine is competent to do so safely

b. Checked that the client intends to use the medicine for a purpose for which it is authorized

c. Advised the client on the safe administration of the medicine and on any warnings/contraindications stated on the label or package leaflet

d. All of the above

4. How can a VPP prepare for an inspection by the VMD?
a. Contact the RCVS to discuss what is required
b. Phone a friend
c. Read the VMD retail of veterinary medicine guidance webpage
d. All of the above 